\title{
Reducing maternal suicide in Sri Lanka: closing the gap
}

\author{
L L A Isuru, K D K Gunathillaka, S T Kathriarachchi
}

Pregnancy and childbirth are usually described as periods of happiness in a woman's life. Unfortunately, it is also a time of increased vulnerability for mental health issues. In addition to the normal physiological changes of pregnancy and puerperium, assumption of the responsibility of motherhood, together with pressure of needing to resume employment contribute to the stress of a new mother. Increased vulnerability to mental disorders may lead to suicide in the perinatal period (1).

Maternal death is defined as the death of a woman while pregnant or within the puerperium (2). Late maternal death includes those that occur within one year after the end of the pregnancy, and if this death occurs due to suicide, it is described as a maternal suicide. The effect of suicide during pregnancy or during the immediate postpartum period is far reaching. Apart from the calamity of the loss of life of a mother, it inflicts significant negative health and socioeconomic impacts on living children, the family and community (1). Recent evidence suggests that maternal suicide can increase suicidal tendencies of offspring in later life (3).

Measures taken in recent years in Sri Lanka to promote a safe pregnancy and puerperium have led to a decline of maternal mortality rate from 500 per 100,000 live births in 1950 's to, 30 per 100,000 in 2015. However, maternal suicide rates have increased from the year 2002 to 2010 (Figure 1). For example, a study conducted in North Central Province found that $17.8 \%$ of recorded maternal deaths were due to suicide, ranking it number one among causes of maternal deaths (4). Of the mothers who died by suicide, $79 \%$ were less than 30 years old, with the highest number of deaths occurring in the 26 - 30 year age group. (1). This increase in maternal suicide in Sri Lanka is in marked contrast to the decrease in rates of maternal mortality (due to other causes) (Figure 2). This data highlights the increasing importance of maternal suicide as a contributor to maternal deaths. Thus, the identification of associated factors and possible methods of prevention of maternal deaths due to suicide, is becoming increasingly important.

Globally, maternal suicides receive inadequate attention (5). In Sri Lanka, the paucity of data regarding the magnitude of this problem is a major drawback in designing effective interventions. Maternal mental health and maternal suicides are understudied in Sri Lanka (6). Directly extrapolating prevention and intervention strategies used in the West may not be suited or applicable to the situation in Sri Lanka.

More information is needed to assess the prevalence of maternal suicide in different localities and to evaluate associated factors and trends of change. International studies indicate that factors associated with maternal suicide are multiple and varied. Investigators of the phenomenon have so far been unable to pinpoint any single factor as the major contributor. In order to elucidate the major contributors in the Sri Lankan setting, better information from epidemiological studies and psychological autopsies are needed.

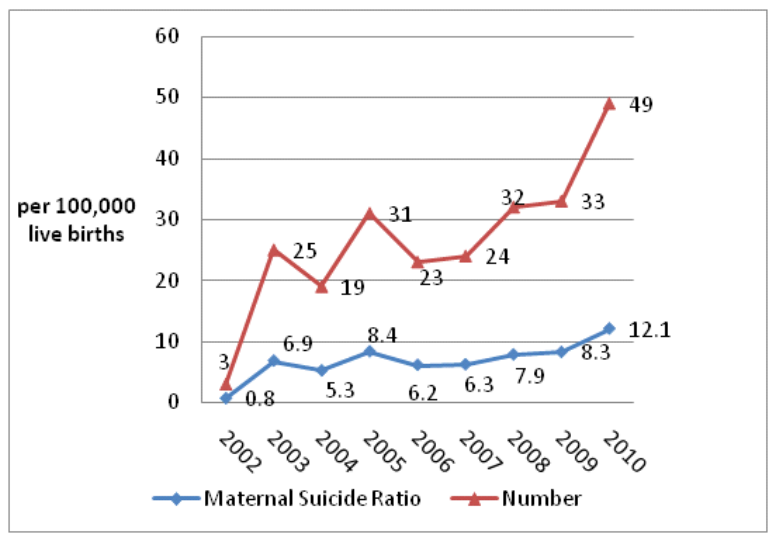

Figure 1. Maternal suicides 2002-2010. Reproduced, with permission from $\mathrm{K}$. Jayaratne.

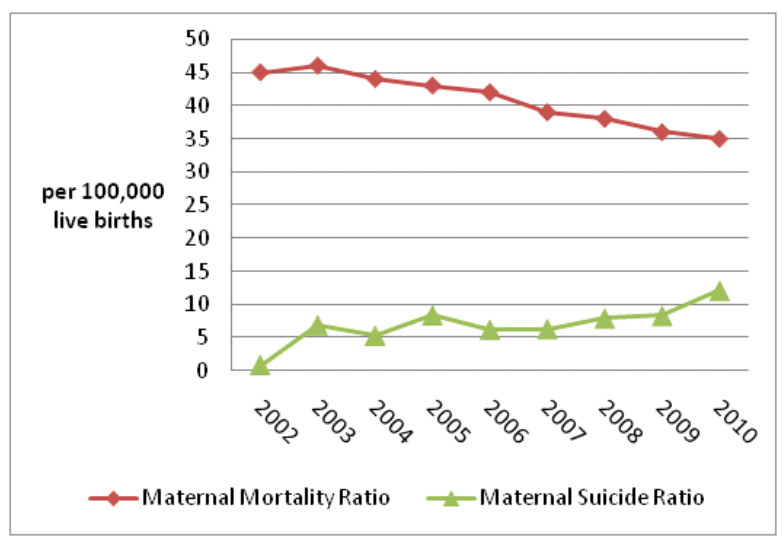

Figure 2. Maternal mortality and maternal suicide ratio 2002-2010.

\section{Is identification of maternal mental illness important in preventing maternal suicide?}

Psychiatric illnesses have been identified as a major cause of maternal suicides across the globe (7). In the UK, a psychiatric diagnosis was identified in $86 \%$ of maternal suicides (7). Up to $4 \%$ of mothers with postpartum psychosis are reported to commit infanticide (8). About 10\% 
of pregnant women and $13 \%$ of postpartum mothers experience a mental disorder, the commonest disorder being depression (9). In developing countries, rates of depression are $15.6 \%$ during pregnancy and 19.8\% after childbirth (9). Sri Lankan data is limited, but according to a study done in the Anuradhapura district, the rate of antenatal depression was $16 \%$ (10), while another study estimated the prevalence of postpartum depression to be around $27 \%$ (11).

A prior history of mental illness is the strongest predictor of perinatal mental disorders (12). Other risk factors described in a recent review were: Having a partner lacking in empathy or who is openly antagonistic, being a victim of gender-based violence, having confrontational in-laws, being socially disadvantaged, having no reproductive autonomy, having an unintended or unwanted pregnancy, having a pregnancy-related illness or disability, lack of support from one's mother, and giving birth to a female infant (6).

Importantly, perinatal mental health disorders, particularly depression, show a good response to treatment $(13,14)$. Early treatment of prenatal and postnatal mental health problems would benefit not only the mother's mental health, but also the infant's physical health and development (13). The importance of multiple biological, psychological and social factors in the causation of maternal mental illness indicates that prevention and treatment programs should be multifaceted (15). By offering timely and appropriate pharmacological, psychological and social interventions, a healthy outcome is more likely for the mother, infant and the family.

\section{Barriers to reducing maternal suicides in Sri Lanka}

Barriers to reduce maternal suicides in Sri Lanka are manifold. Some of these factors include: the paucity of research on prevalence of maternal suicide and associated risk factors, underreporting leading to non identification of cases, lack of awareness and knowledge about psychiatric disorders in the perinatal period, poor integration between mental health services and maternal health services, lack of supervised screening programmes for identification of vulnerable or high risk mothers, and lack of identification of barriers to seek treatment.

Maternal suicides are underreported in Sri Lanka, and so far it has not been considered to be a direct cause of maternal mortality, even when suicide occurs in the context of postpartum psychosis or postpartum depression (7). Maternal suicides would inflate maternal mortality statistics, if included (4). Inclusion of maternal suicide data in maternal mortality statistics will help to identify the mental health gap and will enable to develop sound strategic planning. Similar strategies have led to positive results in many other countries. In Sri Lanka too, the policy being currently developed envisages maternal suicides being recorded as part of maternal mortality data. The proposed system suggests that mortality data of pregnancy and up to one year after termination of pregnancy should be noted within the national maternal mortality surveillance system (16). The deaths are to be analysed and preventive strategies discussed at maternal mortality review meetings. This system, when implemented will provide more accurate data on maternal mortality. It is important that health professionals working in the fields of maternal health and psychiatry should collaborate and work together in this regard, to ensure that psychiatric illness and the risk of suicide is not missed or underestimated, during the perinatal period.

Since 2012, the locally validated Postnatal Edinburgh Depression Scale has been administered to postnatal mothers in Sri Lanka by family health workers, as part of the national pregnancy care program of postpartum care. Despite this, maternal depression is still heavily under diagnosed in the Sri Lankan setting (11). Lack of supervision of the current screening system, and poor integration into the mental health system may contribute to this, and all of which need to be addressed. In addition to screening, case finding by risk factor identification is highly recommended (17). Inquiry into risk factors and psychological well being of pregnant and postpartum mothers should be a part of the assessment carried out by health care professionals involved in maternal care. Members of the multidisciplinary team who may have more information about the individual and their family, should be involved in the management process, in order to aid identification of risk factors in personal as well as family and psychosocial history (12).

Inadequate mental health literacy among health care professionals who interact with pregnant and postpartum mothers is a major drawback in the prevention of maternal suicides. The inclusion of psychiatry as a subject in the medical curriculum is a positive step in this regard. Health care professionals such as general practitioners, medical officers of health, family health workers and obstetrics teams should have a sound awareness of risk factors, symptomatology and common presentations of maternal mental disorders, in order to recognise when to refer for further assessment and care. Detection and adequate management of these disorders require co-ordinated approach among primary care, obstetric teams with local mental health teams (18).

Other factors such as stigma and differing culturally beliefs of the women and families concerned may also contribute to underutilization of available mental health care facilities. The lack of developed community mental health care teams contributes to the difficulty in reaching those mothers who may be reluctant to seek help.

\section{The way forward}

Inclusion of maternal suicides in national statistics as a cause of maternal mortality is an important step forward, in order to correctly portray the present state of the 
problem in Sri Lanka. Together with this, well-designed studies should be conducted to detect the prevalence and risk factors associated with maternal suicide in Sri Lanka, as well as the existing barriers to seeking treatment. Comprehensive, in-depth analysis of each of maternal suicides by use of psychological autopsy, in collaboration with the psychiatrist, is essential to improve our understanding of maternal mental disorders and other circumstances surrounding maternal suicide in Sri Lanka.

Maternal mental health should be integrated into primary, secondary and tertiary care of women of reproductive age. In order to close the knowledge gap in this area, education about perinatal morbidity and mortality from mental illness need to be incorporated into training curricula, continuing professional development programmes, and in-service training of professionals caring for perinatal mothers such as family health workers, medical officers of health, general practitioners and obstetricians. Further to this, screening for maternal mental health disorders should be properly monitored and a protocol should be developed to guide the health care providers to facilitate appropriate referral to mental health care teams.

\section{Conclusions}

Suicide is one of the leading causes of maternal mortality. Maternal suicide is increasing in Sri Lanka. Early identification, referral and management of high-risk mothers is likely to help reduce this tragic outcome. The above recommendations will pave the way to establish an appropriate pathway to detect, refer and manage pregnant and postpartum mothers with mental disorders and those at risk of suicide; this will help reduce the rate of maternal suicide and maternal mortality, and will consolidate the laudable gains in the field of maternal health which have been already achieved in Sri Lanka.

\section{Acknowledgements}

Dr. Kapila Jayaratne, Maternal Mortality Surveillance Unit, Family Health Bureau, Colombo.

\section{Conflicts of interest}

None declared

\section{L A Isuru, Base Hospital Thambuththegama}

K D K Gunathillaka, Faculty of Medicine, University of Colombo

S T Kathriarachchi, Department of Psychiatry, Faculty of Medicine, University of Sri Jayawardhanapura.

Corresponding author: S T Katriarachchi

E-mail: prof.stk@sjp.ac.lk

\section{References}

1. Jayaratne K. Maternal Suicides in Sri Lanka: Lessons learnt from review of maternal deaths over 9 years (2002-2010). In: Suicide in Sri Lanka: Past, Present and Future Transformations. World Health Organization; 2013.

2. World Health Organization. ICD-10 international statistical classification of diseases and related health problems. Geneva: World Health Organization; 2010.

3. Brent DA, Melhem NM, Mann JJ. Pathways to Offspring Suicidal Behavior May Begin With Maternal Suicide Attempt. J Am Acad Child Adolesc Psychiatry. 2015; 54(10): 868.

4. Agampodi S, Wickramage K, Agampodi T, et al. Maternal mortality revisited: the application of the new ICD-MM classification system in reference to maternal deaths in Sri Lanka. Reprod Health. 2014; 11(1): 17.

5. Sahingöz M, Yuksel G, Karsidag C, et al. Birth weight and preterm birth in babies of pregnant women with major depression in relation to treatment with antidepressants: J Clin Psychopharmacol. 2014; 34(2): 226-9.

6. Fisher J, Cabral de Mello M, Patel V, et al. Prevalence and determinants of common perinatal mental disorders in women in low- and lower-middle-income countries: a systematic review. Bull World Health Organ. 2012; 90(2): 139G-149G.

7. Oates M. Suicide: the leading cause of maternal death. $\mathrm{Br}$ J Psychiatry. 2003; 183(4): 279-81.

8. Sadock BJ, Sadock VA, Ruiz P, Kaplan HI, editors. Kaplan \& Sadock's comprehensive textbook of psychiatry. 9th ed. Philadelphia: Wolters Kluwer Health/Lippincott Williams \& Wilkins; 2009. 2 p.

9. Rahman A, Fisher J, Bower P, et al. Interventions for common perinatal mental disorders in women in low- and middle-income countries: a systematic review and metaanalysis. Bull World Health Organ. 2013; 91(8): 593-601I.

10. Agampodi SB, Agampodi TC. Antenatal depression in Anuradhapura, Sri Lanka and the factor structure of the Sinhalese version of Edinburgh postpartum depression scale among pregnant women. PLoS ONE. 2013; 8(7): e69708.

11. Agampodi $\mathrm{T}$, Agampodi S, Wickramasinghe W, Adhikari A, Chathurani H. Post partum depression - a problem that needs urgent attention. Ceylon Med J . 2011; 56(4): 183-4.

12. National Institute of Clinical Excellence [Internet]. Antenatal and postnatal mental health: clinical management and service guidance. Available from: https://www. nice.org.uk/guidance/cg192 (accessed 16 April 2016).

13. Brockington I. Postpartum psychiatric disorders. The Lancet. 2004; 363(9405): 303-10.

14. World Health Organization (WHO). Maternal mental health and child health and development in low and middle income countries: report of the meeting, Geneva, Switzerland, 30 January-1 February, 2008. Geneva: World Health Organization; 2008.

15. Bledsoe SE. Treating depression during pregnancy and the postpartum: a preliminary meta-analysis. Res Soc Work Pract. 2006 Mar 1; 16(2): 109-20.

16. Jayaratne K, Perera D. Surveillance and response system for maternal deaths in Sri Lanka: an audit. The Lancet. 2014; 384: S9.

17. Patrick K. It's time to put maternal suicide under the microscope. Can Med Assoc J. 2013; 185(13): 1115.

18. Royal College of Obstetricians and Gynaecologists. Management of Women with Mental Health Issues during Pregnancy and the Postnatal Period. Good practice No 14. Royal College of Obstetricians and Gynaecologists.; 2011. 